\title{
Modelo de gestión de la energía en la industria de fabricación de harina de trigo
}

\section{Energy management model in the Wheat Flour Manufacturing Industry}

\author{
José Vásquez Araya* \\ *Universidad Técnica Nacional, Costa Rica. Contacto: javasquez@utn.ac.cr
}

Referencia/ reference:

Vásquez, J. (2020). Modelo de gestión de la Energía en la Industria de Fabricación de Harina de Trigo. Yulök Revista de Innovación Académica, 4(1), 48-55.

\section{Resumen}

En este documento se presenta el diseño de un modelo de gestión de la energía basado en la norma INTE/ISO 50001 (2018), para una industria de fabricación de harina de trigo. El desarrollo de un modelo de gestión de la energía, el cual posee un impulso importante en la actualidad nacional, busca mejorar el desempeño energético de los procesos productivos, así como de brindar información requerida, por la Gerencia, para el establecimiento de las directrices referentes a la administración de la energía.

Palabras clave: Indicador de desempeño energético, línea base energética, objetivos y metas energéticas, modelo de gestión de la energía.

\section{Abstract}

This document presents the design of an energy management model based on the INTE / ISO 50001 (2018) standard for a wheat flour manufacturing industry. The development of an energy management model, which has an important momentum in the national situation, seeks to improve the energy efficiency of production processes as well as to provide information required, by management, for the establishment of guidelines related to Energy management.

Keywords: Energy performance indicator, energy baseline, energy goals and targets, energy management model. 


\section{Introducción}

El Plan Nacional de Energía 2015-2030 del MINAE estimula la eficiencia energética de los grandes consumidores. Este Plan, establece como uno de los objetivos, a medio plazo, la adopción de la norma ISO 50001 de Gestión Energética en los macroconsumidores. La industria de la producción de harina de trigo, por su proceso productivo, requiere principalmente del uso de cargas eléctricas cómo motores eléctricos, en distintas gamas de potencia, los cuales son empleados en los molinos, elevadores, bandas, ventiladores, etc. El uso principal de este tipo de carga propicia que posean un consumo significativo de energía y una alta demanda de potencia de la red de suministro eléctrico. El presente documento genera un modelo base, con el cual se puede implementar la gestión de la energía en este tipo de industria, así como en otras industrias con procesos productivos de características similares. El modelo de gestión de energía planteado se basa en la última versión de la norma ISO 50001 (2018), no obstante, se intenta dar un enfoque dentro del entorno nacional y generar ese primer paso para la mejora del desempeño energético en estos tipos de industria.

\section{Modelo de Gestión de la Energía: Contexto, liderazgo y planificación}

El modelo utilizado para el diseño fue el que se establece en la norma INTE/ISO 50001 (2018), el cual se puede apreciar en la figura 1. Después se desarrolló cada uno de los puntos establecidos en dicha normativa adaptándose al contexto existente. En el presente documento se evalúan los principales puntos que aplican de forma general en este tipo de industrias. (figura 1)

La figura anterior muestra el ciclo PHVA de mejora continua, enfocado a la gestión de la energía para un alcance determinado. En este no solamente interviene el accionar de la organización si no también las cuestiones internas y externas, el contexto de la organización y las necesidades y expectativas de las partes interesadas. Todos estos elementos son elementos fundamentales dentro del modelo.

\section{Contexto de la organización}

Se debe desarrollar una comprensión de la organización y su contexto financiero, legal, social, productivo, etc. Por lo que se evalúan las necesidades y expectativas de todos los involucrados en el modelo. Es importante definir el alcance y los límites, se recomienda que los usos

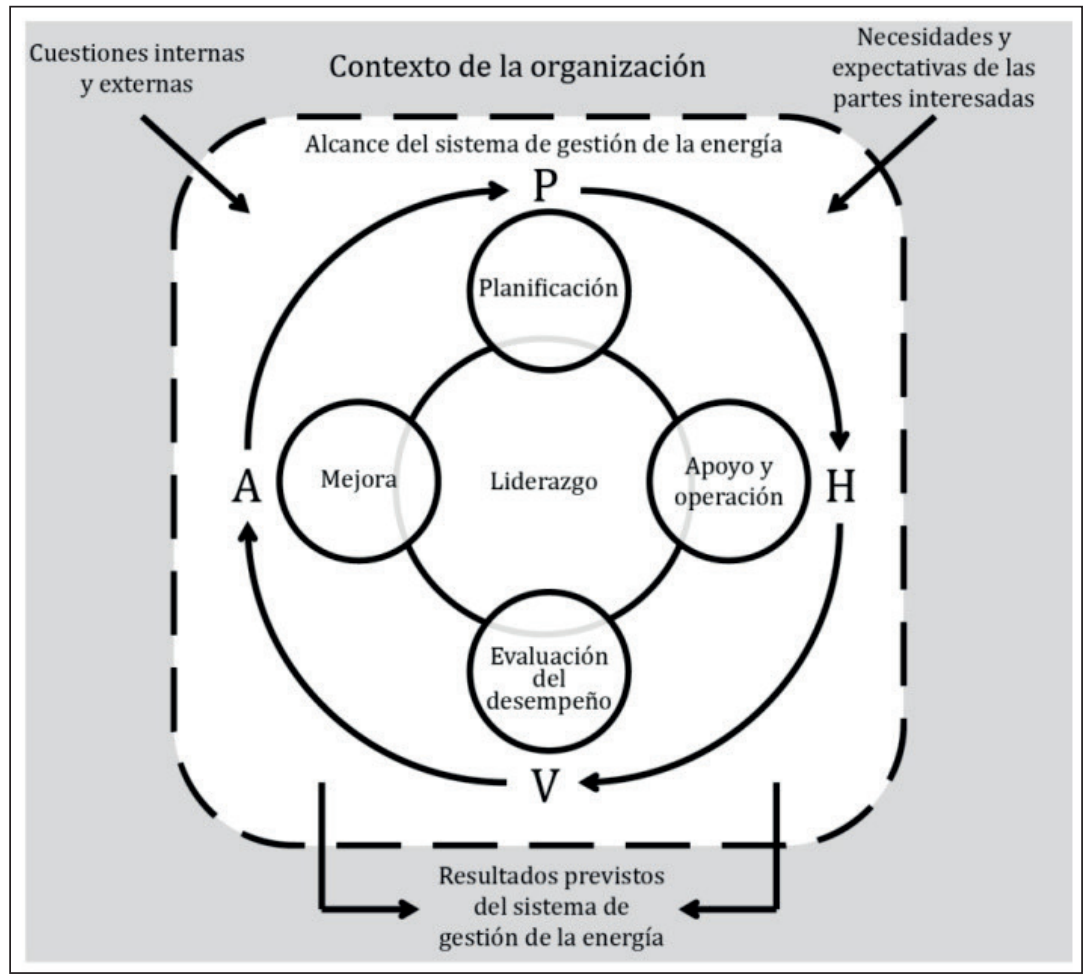

Figura 1. Ciclo Planificar-Hacer-Verificar-Actuar. Fuente: Norma INTE/ISO 50001 (2018). 
de energía sean determinados por proceso productivo, ya que la mayoría del consumo de energía es eléctrico, lo que no permitiría una evaluación correcta en cada una de las partes del proceso productivo. Para este tipo de industria se puede realizar una definición de usos de energía enfocados en los siguientes procesos:

- Recepción y traslado del trigo

- Proceso de limpia del trigo

- Proceso de molienda del trigo

- Proceso de manejo de harina

- Empaque y despacho

- Facilidades

- Procesos de apoyo

\section{Liderazgo}

El liderazgo y compromiso de la Alta Dirección con el modelo de gestión de energía es de vital importancia para el éxito de es. En este punto se debe definir la política energética, en la cual se funda la base del modelo, así como el equipo de gestión de la energía, los cuales son los responsables de llevar el peso del funcionamiento de este. Debe incorporar personal de producción, mantenimiento e ingeniería, calidad y ambiente, compras y recursos humanos.

\section{Planificación}

En este punto se debe evaluar y abordar los riesgos que pueden provocar el no cumplimiento de lo establecido en el modelo con respecto al desempeño energético, así como las oportunidades que se generen de este análisis.

La revisión energética, también, se desarrolla en este punto, siendo uno de los elementos claves para las bases del modelo. Para esta revisión se recomienda la realización de una auditoría energética nivel II, con el fin de cuantificar todos los usos de energía, así como establecer las Oportunidades de Conservación de la Energía que permitan definir objetivos y metas energéticas logrables.

Si no se posee información del consumo de energía por uso de energía (proceso productivo), se debe hacer un análisis de los diagramas unifilares y de la carga instalada, con el fin de establecer el proceso de medición de la auditoría. A continuación, se muestra una herramienta, denominada Diagrama de Pareto de la carga instalada.

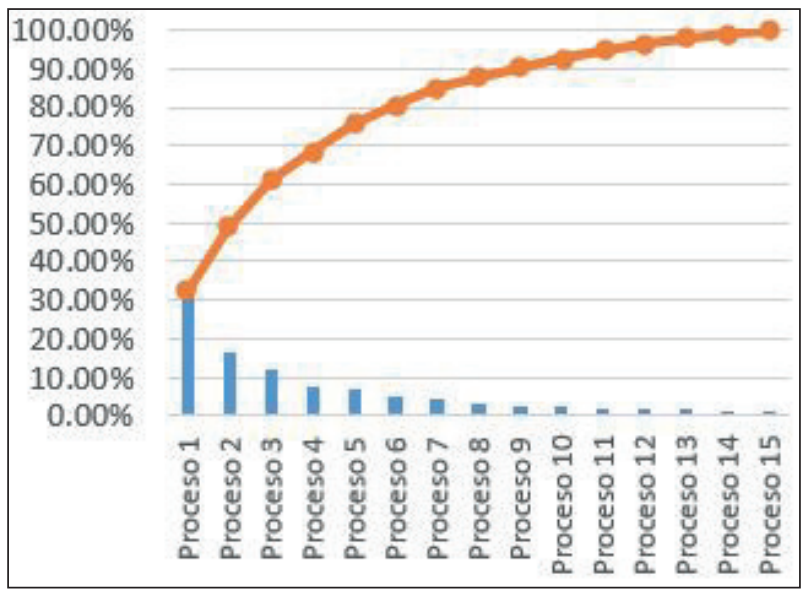

Figura 2. Diagrama de Pareto de Carga Instalada en una industria de harina de trigo. Fuente: elaboración propia, 2020.

Como parte de la auditoría, el análisis de la facturación, con un periodo no menor a un año, puede arrojar información importante sobre si existe control de la demanda en periodos punta, el peso de la energía y la demanda en la facturación, así como comportamientos inusuales o normales con respecto a la producción de la planta productiva. A continuación, se muestra en la figura 3 el costo de energía y demanda para una industria de producción de harina de trigo.

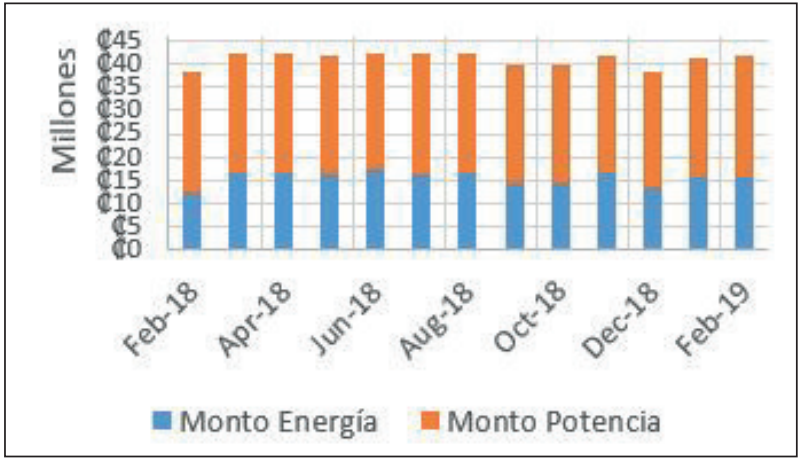

Figura 3. Monto de energía y demanda mensuales para una industria de producción de harina de trigo. Fuente: elaboración propia, 2020.

Para el caso de Tarifa T-MT, que es la existente para la industria de la figura, existe un costo importante en demanda por lo que la gestión de esta en los distintos periodos horarios se considera una importante oportunidad de mejora en la facturación.

Otro procedimiento importante por desarrollar en esta etapa es la de analizar los perfiles de carga de los usos de energía. Con esta información se puede conocer oportu- 
nidades de conservación, desde eliminar malas prácticas de uso de la energía hasta mejoras en el proceso de producción. A continuación, se muestra la figura 4, la cual muestra el comportamiento de un par de compresores durante la operación de una planta de producción de harina de trigo. En ella se muestra en cuales periodos existe una mayor demanda y si está relacionada con la producción.

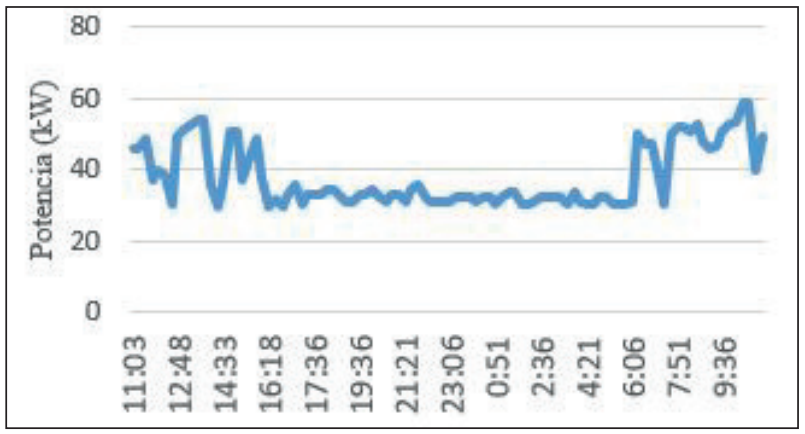

Figura 4. Perfil de carga de sistema de generación de aire comprimido. Fuente: elaboración propia, 2020.

\section{Balance de energía}

Es importante determinar los principales consumos por usos de energía, ya que este es uno de los puntos importantes a tomar en cuenta para lograr definir de forma adecuada los usos significativos de energía (USEs), en los cuales se van a enfocar más los refuerzos para mejorar su desempeño energético. A continuación, se muestra una tabla donde se observa, para distintos procesos, el peso del consumo de energía.

Tabla 1. Balance de energía de industria de producción de harina

\begin{tabular}{|l|c|c|}
\hline Descripción & Energía $(\mathbf{k W h})$ & Porcentaje \\
\hline Proceso 1 & 212308,04 & $45,78 \%$ \\
\hline Proceso 2 & 108882,48 & $23,48 \%$ \\
\hline Proceso 3 & 39407,1 & $8,50 \%$ \\
\hline Proceso 4 & 26860,71 & $5,79 \%$ \\
\hline Proceso 5 & 9533,86 & $2,06 \%$ \\
\hline Proceso 6 & 7399,27 & $1,60 \%$ \\
\hline Proceso 7 & 2190 & $0,47 \%$ \\
\hline Proceso 8 & 1230 & $0,27 \%$ \\
\hline Proceso 9 & 1670,66 & $0,36 \%$ \\
\hline Proceso 10 & 54237,88 & $11,70 \%$ \\
\hline Total & 463720,00 & $100,00 \%$ \\
\hline
\end{tabular}

Fuente: elaboración propia, 2020.

\section{Usos significativos de energía}

"Los usos significativos de energía (USEs) son aquellos que tienen un consumo sustancial de energía y/o que ofrecen un alto potencial de mejora en el desempeño, por lo que son los puntos en los que la organización debe enfocar su gestión". (Agencia de Sostenibilidad Energética, 2018, p. 41).

Para determinar los usos significativos de energía se utiliza una matriz de evaluación, la cual incluye unos criterios y su respectiva rúbrica para evaluar cada uno de los usos de energía. Se incluye un ejemplo de criterios y forma de evaluar.

Tabla 2. Matriz de criterios para determinar USEs

\begin{tabular}{|c|c|c|c|}
\hline \multicolumn{2}{|c|}{$\begin{array}{c}\text { Porcentaje consu- } \\
\text { mo de energía }\end{array}$} & \multicolumn{2}{c|}{$\begin{array}{c}\text { Potencial de mejora del } \\
\text { desempeño energético }\end{array}$} \\
\hline Criterio & $\begin{array}{c}\text { Evalua- } \\
\text { ción }\end{array}$ & Criterio & Evaluación \\
\hline$>20 \%$ & 3 & Alto & 3 \\
\hline $\begin{array}{c}20 \% \\
-5 \%\end{array}$ & 2 & Medio & 2 \\
\hline$<5 \%$ & 1 & Bajo & 1 \\
\hline
\end{tabular}

Fuente: elaboración propia, 2020.

\section{Línea base energética}

Uno de los elementos más importantes por determinar dentro del diseño del modelo de gestión de la energía es la línea base energética. En la industria de producción de harina de trigo, normalmente, los indicadores de producción no se relacionan con el producto terminado, en este caso la harina, si no que se relaciona con el trigo procesado. Adicional, es importante mencionar que del trigo la cascarilla y otros elementos son aprovechados y se comercializan, por lo que existe un consumo de energía para estos elementos del trigo. Por esta razón se plantea una línea base tomando en cuenta un método de regresión lineal múltiple, donde las variables independientes son el consumo de trigo por clase, y la variable dependiente el consumo de energía eléctrica. A partir de este análisis se 
puede obtener una ecuación que corresponde a la línea base.

A continuación, se muestra en la figura 5 una gráfica donde su modela el consumo calculado versus el real.

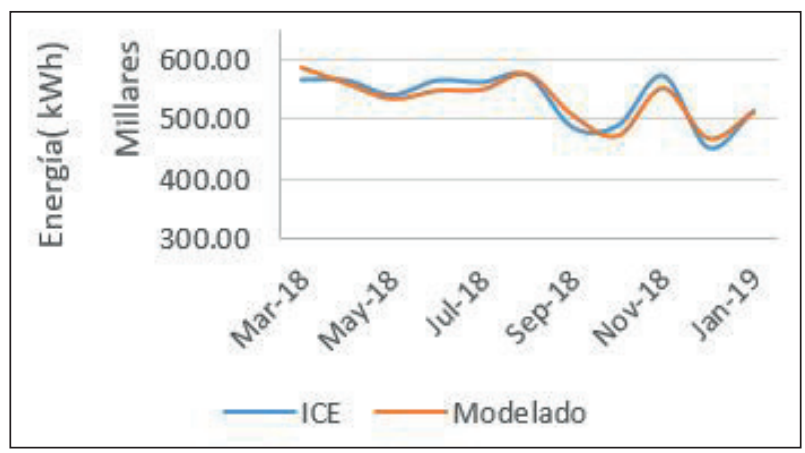

Figura 5. Comparación de consumo de energía facturado por el Instituto Costarricense de Electricidad y el consumo de energía de acuerdo con el modelo de regresión lineal múltiple. Fuente: elaboración propia, 2020.

\section{Indicadores de desempeño energético}

Los indicadores (IDEn) se utilizan para verificar el desempeño energético de la organización. La comparación del comportamiento de un determinado IDE durante el periodo de línea de base y después del inicio del funcionamiento del Sistema de Gestión de Energía puede determinar, por ejemplo, si las acciones implementadas están produciendo los resultados esperados en términos de mejora del desempeño energético o llamar la atención al hecho de que las mejoras de desempeño energético esperadas no se estén alcanzando (Agencia de Sostenibilidad Energética, 2018, p. 44).

La determinación del indicador de desempeño energético se estableció mediante un índice global de producción. De acuerdo con lo establecido del apartado anterior, se analizó la información a través de la variable independiente (consumo de trigo) y la dependiente (consumo de energía), por lo que se seleccionó el de consumo específico de energía eléctrica por consumo de trigo global. A continuación, se muestra la ecuación establecida en el indicador.

$$
C E E P G=\frac{\text { Energía consumida }(k W h)}{\text { Consumo de trigo }(t)}
$$

Una vez definido el indicador, este se puede comparar con los distintos periodos, a continuación, se muestra la figura 6 donde se puede observar esta comparativa.

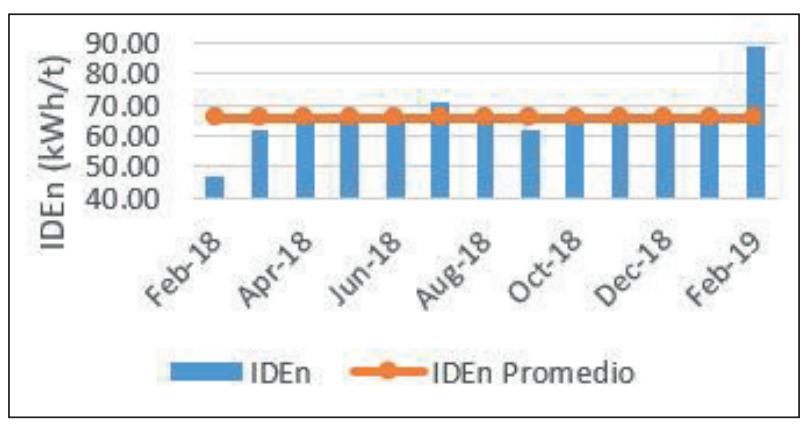

Figura 6. IDEn mensuales reales en comparación con el IDEn promedio calculado. Fuente: elaboración propia, 2020.

Oportunidades de conservación, proyectos de eficiencia energética y mejora de facturación

A continuación, se van a numerar oportunidades, proyectos de eficiencia y de mejora en facturación que se pueden aplicar en la industria de producción de harina de trigo:

Oportunidades de conservación de la energía

- Fugas de aire comprimido.

- Bajar presión de operación en compresores.

- Eliminar malas prácticas de uso de aire comprimido.

- Evitar operación de procesos en vacío

- Ajuste correcto de los cilindros de molienda.

Proyectos de eficiencia energética

- Cambio de motores con mayor eficiencia y carga demandada ajustada.

Proyectos de mejora de facturación

- Cambio de tarifa (Mediante la certificación de un sistema de gestión de la energía con base en la INTE/ ISO 50001, 2018).

- Control de la demanda en periodos horario

Objetivos, metas y planes de acción del modelo de gestión

"La organización deberá establecer objetivos y metas energéticas que tengan la finalidad de mejorar el desempeño energético de la organización. Los objetivos deberán ser documentados y, además, contar con el detalle necesario para asegurar que se alcanzan en los plazos 
definidos." (Agencia de Sostenibilidad Energética, 2018, p. 47)

El establecimiento de los objetivos y metas debe ir acorde con los resultados de la revisión energética, ya que estos deben ser alcanzables, por los que son respaldados de forma técnica. A continuación, se muestran ejemplos de objetivos y metas energéticas:

Tabla 3. Objetivos y metas energéticas.

\begin{tabular}{|c|c|c|}
\hline $\mathbf{N}^{\circ}$ & Objetivo & Meta \\
\hline 1 & $\begin{array}{l}\text { Implementar siste- } \\
\text { ma de medición de } \\
\text { los usos de energía }\end{array}$ & $\begin{array}{l}\text { Instalar a la totalidad } \\
\text { de usos de energía } \\
\text { equipos de medición } \\
\text { junto con sistema de } \\
\text { recopilación y registro }\end{array}$ \\
\hline 2 & $\begin{array}{l}\text { Realizar el levan- } \\
\text { tamiento de la efi- } \\
\text { ciencia y cálculo } \\
\text { de factor de carga } \\
\text { para los motores } \\
\text { del proceso pro- } \\
\text { ductivo }\end{array}$ & $\begin{array}{l}\text { Completar el levan- } \\
\text { tamiento y el cálculo } \\
\text { para el } 100 \% \text { de los } \\
\text { motores del proceso } \\
\text { productivo }\end{array}$ \\
\hline 3 & $\begin{array}{l}\text { Reducir el con- } \\
\text { sumo de energía } \\
\text { eléctrica }\end{array}$ & $\begin{array}{l}\text { Reducir en un por- } \\
\text { centaje el IDEn de } \\
\text { consumo de energía } \\
\text { eléctrica por consumo } \\
\text { de trigo }\end{array}$ \\
\hline 4 & $\begin{array}{l}\text { Reducir la deman- } \\
\text { da en los periodos } \\
\text { punta }\end{array}$ & $\begin{array}{l}\text { Reducir en un porcen- } \\
\text { taje la demanda en el } \\
\text { periodo punta }\end{array}$ \\
\hline 5 & $\begin{array}{l}\text { Reducir el con- } \\
\text { sumo de energía } \\
\text { eléctrica para ge- } \\
\text { neración de aire } \\
\text { comprimido }\end{array}$ & $\begin{array}{l}\text { Reducir un porcentaje } \\
\text { el consumo de energía } \\
\text { eléctrica para la gene- } \\
\text { ración de aire compri- } \\
\text { mido }\end{array}$ \\
\hline 6 & $\begin{array}{l}\text { Sensibilizar al } \\
\text { personal de la em- } \\
\text { presa con respecto } \\
\text { a la gestión de la } \\
\text { energía }\end{array}$ & $\begin{array}{l}\text { La toma de concien- } \\
\text { cia de la gestión de la } \\
\text { energía por parte del } \\
\text { personal, con la elimi- } \\
\text { nación de tres malas } \\
\text { prácticas del uso de la } \\
\text { energía }\end{array}$ \\
\hline
\end{tabular}

Fuente: elaboración propia, 2020.
Cabe destacar que los objetivos dados de ejemplos deber ser respaldados por una correcta planificación para poder ser alcanzados, donde se defina que se va a hacer, qué recursos serán necesarios, los responsables y la programación y evaluación del cumplimiento.

\section{Planificación para la recopilación de datos de la energía}

Unos de los puntos claves en el éxito del modelo de la gestión de la energía, es la correcta medición del consumo de energía. Dependiendo del tipo de energía se debe seleccionar el equipo de medición, no obstante, se debe establecer el plan para medir, registrar y revisar la información.

\section{Apoyo}

La organización debe proporcionar el recurso humano financiero, recursos tecnológicos y de equipamiento para la medición y registro de los datos sobre el desempeño energético para que el modelo de gestión se pueda implementar. Adicional, se debe elaborar un plan de capacitación de la empresa de acuerdo con las necesidades del personal, en función del perfil y el rol que posea cada quien, dentro de la organización, esto para que el colaborador desempeñe su cargo con responsabilidad sobre el uso de la energía. Es importante que el personal tome conciencia sobre la política energética, el equipo de gestión de energía, los USEs, los objetivos y las metas planteadas, los beneficios en la mejora del desempeño y la responsabilidad por el incumplimiento de lo establecido en el modelo de gestión. La organización debe tomar la decisión de comunicar la información respecto al modelo de gestión de la energía, y si lo hace debe determinar cuándo y que tipo de información, así como los responsables y los medios de hacerlo. Esto aplica tanto para lo interno como lo externo de la empresa.

\section{Operación}

El definir los controles operacionales es de suma importancia para el modelo de gestión y la mejora del desempeño energético de la organización.

Para un modelo de gestión de la energía en industria de fabricación de harina de trigo, se considera que se deben implementar los siguientes controles operacionales: 
1. Mantenimiento y rectificación de los rodillos de los molinos de la molienda.

2. Ajuste de los rodillos de los molinos de la molienda.

3. Registro del sistema de medición del modelo de gestión.

4. Registro de tiempos no asociados con producción por los usos de energía.

5. Control de manejo o desplazamiento de carga definidos.

6. Control de fugas de la red de aire comprimido.

7. Mantenimiento de motores basado en revisión del nivel de tensión, alineamiento, vibración, termografía y lubricación.

También, es importante mencionar en esta etapa, que cualquier diseño de una mejora o ampliación en las instalaciones de la planta, se debe tener como criterio la eficiencia y el uso de la energía, ya que esto podría constituir una oportunidad de mejora del desempeño energético. Adicional, se deben establecer criterios para evaluar la compra de equipos, productos o servicios por parte de la empresa, los cuales pueden afectar su desempeño energético. Debe existir una coordinación entre el departamento que solicita el servicio, equipo o producto y el Departamento de Compras, ya que las especificaciones deben cumplir previamente con los criterios establecidos y deben respetarse durante todo el proceso de compra.

\section{Evaluación del desempeño}

Se debe realizar el seguimiento, la medición y el análisis del desempeño energético para determinar la eficacia de los planes de acción, así como el comportamiento de consumo de energía con respecto a la línea base energética y los indicadores energéticos. Este seguimiento se debe realizar de forma trimestral y anual. Si durante las revisiones se encuentran desviaciones importantes del desempeño energético, se deberá investigar y determinarla.

La empresa deberá realizar auditorías internas, de periodicidad anual, para proporcionar información sobre el desempeño energético y el funcionamiento del modelo de gestión de la energía. La organización debe definir lo siguiente:

- Programa de auditorías.

- Criterio y alcance de las auditorías.

- Selección del equipo auditor.

- Asegurar el correcto flujo de información de los resultados de la auditoría.

- Tomar las acciones requeridas de acuerdo con los resultados de la auditoría.

Es importante el involucramiento de la Alta Dirección. Esta debe revisar anualmente el funcionamiento del modelo de gestión de energía, de acuerdo con los lineamientos establecidos. Esta revisión debe considerar lo siguiente:

- Revisiones previas realizadas.

- Política energética, objetivos, metas e indicadores energéticos.

- Análisis de las oportunidades y riesgos del modelo de gestión de la energía.

- Información sobre el desempeño del modelo, de acuerdo con los seguimientos y resultados de auditorías.

- Oportunidades de mejora del desempeño energético y proyectos de eficiencia energética.

\section{Conclusiones}

Mediante el modelo de gestión de la energía se puede obtener la línea base y los indicadores de desempeño energético para una industria de fabricación de harina de trigo.

Mediante la gestión de la demanda en periodos horarios, se pueden obtener ahorros importantes en la facturación eléctrica.

Se puede determinar un balance de energía que permita conocer los usos de energía más significativos de su proceso.

Existen oportunidades de conservación de la energía importantes en el proceso de fabricación de harina de trigo.

Mediante un modelo de regresión lineal múltiple se puede obtener una línea base y una estimación del consumo de energía independiente de la cantidad de distintas clases de trigo dentro del proceso.

\section{Recomendaciones}

Se debe realizar una evaluación de las redes de distribución de aire comprimido, ya que pueden existir caídas de presión excesivas en la red, esto incide en una presión de trabajo superior para los compresores. 
Se debe realizar la corrección de las fugas de aire comprimido presentes en las redes de distribución de la planta productiva, ya que esto se convierte en un costo importante de la operación de los compresores desde el punto de vista energético y de mantenimiento.

Se debe instalar un sistema de medición, que permita monitorear el comportamiento del desempeño energético de los diferentes usos de energía para la planta productiva.

Se deben registrar todos los movimientos productivos que posean un consumo de energía y afecten el desempeño energético de la organización.

Se debe generar toma de conciencia y las competencias necesarias para el personal que influya en el desempeño energético de la empresa.

Se debe realizar un levantamiento de las eficiencias y factores de carga de todos los motores de la planta, para implementar una estrategia de cambio, ya sea por fallo o por proyectos de eficiencia energética.

\section{Referencias}

Sostenibilidad y eficiencia: el futuro de la energía en Costa Rica. Estado de la Nación 275, contribución especial.

VII Plan Nacional de Energía 2015-2030. Ministerio de Ambiente y Energía MINAE; Programa de las Naciones Unidas para el desarrollo PNUD.
Decreto 40509 - MINAE - MTSS, Plan Intersectorial para el establecimiento de una tarifa eléctrica preferencial para la conservación y mejora del empleo en empresas electro-intensivas.

INTE/ISO 50001 (2018). Sistemas de gestión de la energía-Requisitos con orientación para su uso. https:// www.iso.org/obp/ui/\#iso:std:iso:50001:ed-1:v1:es

Norma INTE/ISO 50002 (2018). Auditorías Energéticas-Requisitos con orientación para su uso. https:// www.iso.org/obp/ui/\#iso:std:iso:50001:ed-1:v1:es

Comisión Nacional para el Uso Eficiente de la Energía (CONUE). (2018). Manual para la implementación de un sistema de gestión de la energía en el contexto centroamericano. México. ASI/CADIN/ Conuee/ GIZ.

Agencia de Sostenibilidad Energética. (2018). Guía de implementación de Sistemas de Gestión de Energía basados en ISO 50001.

Cooperación Alemana-ICE. Buenas Prácticas de Eficiencia Energética para Motores Eléctricos Industriales. Costa Rica.

FIDE-CNEE. (2018). Curso-Taller Promotores de Ahorro y Eficiencia de Energía Eléctrica. Guatemala.

Programa Estado de la Nación, 2010. Decimosexto Informe Estado de la Nación en Desarrollo Humano Sostenible. Programa Estado de la Nación. 